\title{
Mutations affecting self-incompatibility in Phalaris coerulescens Desf. (Poaceae)
}

\author{
D. L. HAYMAN \& J. RICHTER \\ Department of Genetics, University of Adelaide, South Australia 5001, Australia
}

\begin{abstract}
A total of 508 possible mutants affecting self-incompatibility in Phalaris coerulescens were obtained from a mutant trap experiment. None of these 'mutants', included mutations to a new functional incompatibility allele and 356 behaved as revertants. One hundred and fifty-two of the mutants were self-compatible and the methods of analysis of these mutants are described and the results from studying 37 of them are reported. Sixteen are mutants of the $S$ locus including pollen-only mutants and one complete mutant; six are pollen-only mutants at the $Z$ locus and 16 are mutants at a further locus $T$. This locus does not act to provide genetic diversity in specifying the incompatibility status of the pollen or style. Possible roles for this locus are suggested.
\end{abstract}

Keywords: incompatibility, mutation, Phalaris, Poaceae.

\section{Introduction}

Incompatibility in flowering plants is a genetically determined system that regulates outbreeding. A number of different systems of genetic control have been described, and it is usual for members of the same taxonomic family to have the same system. In the Poaceae, genetic determination of incompatibility is under the control of two unlinked genes, $S$ and $Z$, each with a series of alleles. A pollination is compatible if both the alleles present in the pollen are matched in the style. The incompatibility specification is determined by complementary interaction and is gametophytic in expression in the pollen. The genes act independently in the style. In an autotetraploid the same complementary action occurs: a pollen grain is incompatible if any two of the non-allelic genes present in the pollen are present in a style.

The system of genetic control present in the Poaceae differs substantially from the single locus gametophytic system commonly present in homomorphic dicotyledons, both in the number of loci involved in the determination of specificity and in the interaction of non-allelic genes in diploid pollen. In order to increase the understanding of the Poaceae system an intensive study has been made of mutants affecting incompatibility.

Corresponding author: Dr D. L. Hayman, Department of Genetics, University of Adelaide, GPO Box 498, Adelaide, South Australia 5001 .

\section{Materials and methods}

\section{The choice of species}

The species chosen for this study was the diploid perennial grass Phalaris coerulescens. The grass is highly self-incompatible and is a native of the Mediterranean region. The plant is capable of extensive clonal propagation. So far as is known, the plants grown in this study are the only plants of this species grown in South Australia. Thus there was no possibility of foreign' pollen and the mutant trap described later should have yielded only mutant progeny. The plants flower in October in the glasshouse and field, and a reduced second flowering occurs in the glasshouse in February. Stigmas free of self-pollen may be dissected out readily from unopened florets.

The compatibility status of pollinations was determined by pollinating dissected stigmas which were planted in dishes of 2 per cent agar, 10 per cent sucrose, 100 p.p.m. boric acid (Lundqvist, 1961). These were left in a moist atmosphere overnight at $25^{\circ} \mathrm{C}$ and then stained with cotton blue and lactophenol (Watkins, 1925) and examined $24 \mathrm{~h}$ later. The appearance of compatible and incompatible pollinators has been described previously (Hayman, 1956), and the different degrees of compatibility, as described in that paper, were determined by microscopic examination.

Genetic stocks of known incompatibility status to be used as testers were constructed by identifying two 
closely related plants which were compatible in one direction only. The genetics of the system requires that one of these is heterozygous at both loci, i.e. $S_{12} Z_{12}$. The other is homozygous at one locus and heterozygous at the other and has all incompatibility alleles in common with the first, e.g. $S_{12} Z_{11}$. The progeny from crossing these plants are $S_{11} Z_{12}, S_{22} Z_{12}$ and $S_{12} Z_{12}$. These may be differentiated by pollination tests. The remaining tester genotype $S_{12} Z_{22}$ is obtained after crossing either $S_{11} Z_{12}$ or $S_{22} Z_{12}$ with $S_{12} Z_{12}$ pollen. Thus, apart from the double heterozygote all the other tester genotypes depend upon the arbitrary allocation of the initial genotype $S_{12} Z_{11}$ and this determines which plants are nominated as $S$ homozygotes and $Z$ homozygotes.

\section{The recovery of mutants}

More than 300 plants derived from crossing $S_{12} Z_{11}$ with $S_{12} Z_{12}$ were interplanted in the field with clonal offsets of $S_{12} Z_{12}$. The plants were allowed to self and to interpollinate and heads were then bagged on $S_{12} Z_{12}$ plants. This procedure constituted a mutant 'trap' because unless there was a change in the incompatibility system these heads would contain no seeds.

Heads were harvested in groups of approximately 25 . Seeds were recovered at an average of four per 25 heads. Samples of typical heads, which varied greatly in size, were dissected out and each contained an average of 200 perfect florets. Open pollinated stigmas had an average of 200 pollen grains on the stigmatic branches. Again there was considerable variation in this figure; in addition, crowding of the pollen grains on a stigma interferes with pollen grain germination so that not all pollen grains are equally likely to effect pollination. Thus, the ratio of 1 in 250,000, which is an estimate of the actual seed set compared to the potential seed set, is crude. The maximum number of seeds obtained from any sample of 25 heads was 30 , and the distribution of seeds recovered from all samples suggested that there was no substantial contribution from single events, such as large 'mutant' sectors in any single head.

Seedlings grown from these 'mutant' seeds were planted in the field: each plant adjacent to an offset of $S_{12} Z_{12}$. These plants were selfed and crossed with $S_{12} Z_{12}$

One hundred and fifty-two of these plants were selfcompatible and 356 were self-incompatible. No selfincompatible plant gave seeds as both male and female parents on crossing with $S_{12} Z_{12}$. Thus there is no evidence of a mutation to a new fully functional incompatibility gene.

Some 60 self-compatible plants were selected for further study. Usually only one plant was chosen from the seedlings originating from each original set of bagged heads to reduce the likelihood of recovering the same mutant twice. These plants were brought into the glasshouse and selfed and crossed reciprocally with the testers to characterize the stigmatic and pollen grain behaviour. Meiosis was examined in each plant and no evidence was found of gross structural or numerical chromosome aberrations.

The nature of the experiment results in an intense degree of inbreeding in this normally outbred material. The effects of this inbreeding were seen in the occurrence of univalents at meiosis in some plants, and in varying degrees of male infertility largely due either to a failure to form anthers, which exerted, or to form anthers, which freely shed quantities of viable pollen. This latter factor restricted the number of plants in which the basis of self-compatibility could be analysed. It also limited the recovery of analysable plants possibly homozygous for self-compatibility.

All plants which freely shed pollen were found to have approximately equal numbers of compatible and incompatible pollen grains of selfed stigmas. In the course of the experiments many mutants were crossed to self-incompatible plants and self-compatibility segregated in a regular Mendelian fashion. These results indicate that the self-compatibility observed was heritable, gametophytic in action and probably due to gene mutation.

\section{The determination of the mutational change}

There are different types of change, which would result in seeds being found in heads from $S_{12} Z_{12}$ plants. One of these is a change at one or other of the incompatibility loci to give a new fully functional allele not represented in the style. Another possible change is that in which a defect occurs in the specification of the pollen grain or the stylar tissue which allows a pollen grain to pass the incompatibility barrier. The studies of Lundqvist $(1962,1968)$ demonstrated that self-fertile mutants of Secale cereale may result from a loss of pollen specificity only, the mutant being fully functional in the style. Such mutations have also been identified in single locus gametophytic systems (e.g. Lewis, 1951). By analogy with the other types of mutant that have been reported in these latter systems, changes affecting stigma specificity only, or changes in which both stylar and pollen specificity are lost, might also be expected.

The mutant trap should readily recover all these mutant types with the exception of those which affect the stigmatic incompatibility only. As mutants were obtained from harvesting seeds from $S_{12} Z_{12}$, the unchanged pollen specificity of any mutant $S$ or $Z$ gene would prevent the pollen carrying a stigma-only mutant 
from effecting fertilization. However, such stigma-only mutants could be recovered if they were also associated with a temporary loss of pollen specificity which allowed the pollen to effect fertilization. Such a model was proposed by Lewis \& Crowe (1953) to account for the recovery of stigma-only mutants in Prunus.

Finally, there remains the possibility that self-fertility may result from changes at another locus or loci. This possibility was suggested by Lundqvist (1968) who could not specifically identify such mutants in his study but who suspected their occurrence.

The presence of two known incompatibility loci and the theoretical possibility of more, combined with the different possibilities of the mutational change resulting in self-compatibility, makes the analysis of these mutants complex. A series of different tests is required to distinguish and identify the mutational change.

An analysis of the ratio of compatible to incompatible pollen in cross pollinations with the testers provides one such test. However, this test is not informative when applied to plants in which the stigma $S$ and $Z$ phenotype suggests that the plants are homozygous at both loci: i.e. $S_{11} Z_{11}, S_{11} Z_{22}, S_{22} Z_{11}$, $S_{22} Z_{22}$. When such plants contain a self-compatible mutant they do not provide discriminatory ratios when crossed as pollen parents to the testers.

The other possible genotypes in this population may be analysed for each of the possible mutant types by crossing with the testers, although, as the argument presented below will show, not all of them will be equally informative.

Mutation at $\mathrm{S}$ or $\mathrm{Z}$ which affect stylar specificity alone. In plants containing such a mutant, the stylar specificity is less extensive than the pollen specificity. For example, a self-compatible plant which behaves as apparently $S_{11} Z_{12}$ when used as a female parent and $S_{12} Z_{12}$ when used as a pollen parent would have an $S_{2}$

Table 1 The expected percentage of compatible pollen observed when pollen-only mutants at $S$ or $Z$ are used as pollen parents against the testers. An asterisk indicates the mutant allele

\begin{tabular}{lccccc}
\hline Mutant & $S_{12} Z_{12}$ & $S_{11} Z_{12}$ & $S_{22} Z_{12}$ & $S_{12} Z_{11}$ & $S_{12} Z_{22}$ \\
\hline \multicolumn{7}{l}{$\begin{array}{l}\text { Stylar genotype is } S_{12} Z_{12} \\
S_{12}^{*} Z_{12}\end{array} 50$} & 100 & 50 & 75 & 75 \\
$S_{12} Z_{12}^{*}$ & 50 & 75 & 75 & 100 & 50 \\
\multicolumn{7}{l}{ Stylar genotype is homozygous at one locus, e.g. $S_{12} Z_{11}$} \\
$S_{12} Z_{11}^{*}$ & 50 & 75 & 75 & 50 & 100 \\
$S_{12}^{*} Z_{11}$ & 50 & 100 & 50 & 50 & 100 \\
$S_{12}^{*} Z_{11}$ & 50 & 50 & 100 & 50 & 100 \\
\hline
\end{tabular}

stigma-only mutant. It follows that an $S_{2}$ stigma-only mutant would not be detected in a plant that behaves as $S_{22} Z_{12}$ is specifying pollen; such a plant would be selfincompatible because it would contain one normal $S_{2}$ gene. Heterozygosity at the relevant locus is required to detect and identify stigma-only mutants.

Mutations at $\mathrm{S}$ or $\mathrm{Z}$ which affect pollen specificity only. Such mutants are immediately recognizable when they occur in plants with a stylar specificity $S_{12} Z_{12}$ [see Table 1]. The pollen specificity in such plants suggests that the plant is homozygous at one locus and this immediately identifies the mutant. The results of crossing plants which are homozygous at one of the two loci and contain such a pollen-only mutant are shown in Table 1. Pollen-only mutants at the heterozygous locus are identifiable; such mutants at the homozygous locus are not, as they may be confused with mutants discussed below.

Mutations at $\mathrm{S}$ or $\mathrm{Z}$ which affect both pollen and stigma specificity, i.e. complete mutants. Plants containing such a mutation would appear to be homozygous for the locus concerned when tested for both pollen and stigma specificities. Crossing complete mutants against the testers does not distinguish them from mutants of the pollen-only mutant.

Mutations at another locus independently segregating from $\mathrm{S}$ or $\mathrm{Z}$. In theory, for reasons which are discussed later, such self-compatible mutants could be complete or pollen-only. A distinction between these two possibilities cannot be made from the ratio of compatible to incompatible pollen grains on crossing to the testers.

Only one genotype at the $S$ and $Z$ loci is discriminatory for mutants at a third locus. This is a plant which is heterozygous at both $S$ and $Z$. The expected ratios of compatible to incompatible pollen, when reciprocally crossed with the testers, are shown in Table 2. The interpretation is unequivocal.

When genotypes which are homozygous at either the $S$ of $Z$ locus and carry a mutation at a third independently segregating locus are crossed as pollen parents with the testers, they give the results shown in Table 2. It is apparent that such a mutant gives identical results to those obtained in the cross illustrated in Table 1 involving the genotype $S_{12} Z_{11}$. The two groups of mutants cannot be distinguished by this test.

In order to clarify the nature of the mutational change additional tests are necessary to cope with all the genotypes obtained in the mutant trap. Obtaining and testing homozygotes for a mutation allows discrimination between the types of mutant, i.e. whether it is complete or not, and may allow determination of the 
Table 2 The expected percentage of compatible pollen obtained when plants having a mutant at a third unlinked locus $(T)$ are reciprocally intercrossed to the testers. For reasons discussed in the text, the plants are assumed to have been homozygous at the $T$ locus before the mutation. Pollen carrying the mutation is compatible on any stigma

\begin{tabular}{|c|c|c|c|c|}
\hline$S_{12} Z_{12} T_{11}$ & $S_{11} Z_{12} T_{11}$ & $S_{22} Z_{12} T_{11}$ & $S_{12} Z_{11} T_{11}$ & $S_{12} Z_{22} T_{11}$ \\
\hline \multicolumn{5}{|c|}{ Genotype $S_{12} Z_{12} T_{11}^{*}\left(T_{1}^{*}\right.$ is mutant $)$} \\
\hline $\mathrm{X}$ mutant 50 & 75 & 75 & 75 & 75 \\
\hline Mutant X & 0 & 0 & 0 & 0 \\
\hline \multicolumn{5}{|c|}{ Genotype $S_{12} Z_{11} T_{11}^{*}\left(T_{1}^{*}\right.$ is mutant $)$} \\
\hline $\mathrm{X}$ mutant 50 & 75 & 75 & 50 & 100 \\
\hline Mutant X 50 & 50 & 50 & 0 & 100 \\
\hline
\end{tabular}

mutant gene. A plant homozygous for a complete mutant will be 100 per cent compatible when used as either a male or female parent and crossed to all testers. A pollen-only mutant would be 100 per cent compatible with all testers when used as a pollen source but not when used as a female; and a stigmaonly mutant would always be 100 per cent compatible when used as a female parent against all tester males.

A third test which may be used to discriminate between mutants at the $S$ and $Z$ loci, and possibly other loci, depends upon linkage between an incompatibility locus and another marker gene. In an appropriate cross or selfing, such linkages may lead to disturbed segregations from which the recomination frequency may be estimated. Consider an $S^{*}$ mutant conferring selfcompatibility which is linked to marker, ' $a$ ' $\frac{S^{*}-a}{S_{2}-A} \frac{Z_{1}}{Z_{2}}$

On selfing, the genotype $A A, A a$ and $a a$ will be found in the frequency $(r / 2),(1 / 2)$ and $(1-r / 2)$ where $r$ is the recombination percentage. Common linkage to a known marker will identify groups of possible allelic mutants.

Finally, mutants may be tested for allelism. A degree of cross pollination occurs when self-compatible heads of $P$. coerulescens are bagged together. Crosses may be detected by using isozyme variants to identify the pollen source. Crosses between allelic mutants should be 100 per cent self-compatible on selfing and crosses between unlinked non-allelic mutants should be 75 per cent compatible on selfing. Depending upon the $S$ and $Z$ genotypes of a double mutant, one of which is a mutant at a third locus, an unusual ratio of 87.5 per cent compatible pollen may be obtained after crossing the double mutant as a male parent to the testers (see Table 3).
Where non-allelic genes are closely linked, the detection of the rare recombinant incompatible pollen grains, as distinct from pollen grains which fail to germinate, could present technical difficulties and they may well not be recognized.

\section{Results}

The stigmatic incompatibility phenotype of selfcompatible plants was determined using pollen from the tester plants. The results are shown in Table 4. A complete analysis of both the pollen and stigmatic phenotypes is only possible in plants which freely shed viable pollen. Consequently, a number of these plants and of possible homozygotes obtained from selfing mutants could not be analysed further.

\section{Identification of $\mathrm{S}$ mutants}

Pollen-only $S$ mutants could be identified in some genotypes by determining the ratio of compatible pollen in crosses with the testers (see Table 1 for example). An additional test for $S$ mutants made use of the observation that the locus of phosphoglucoisomerase PGI-2 (GPI-2) E.5.1.3.9 is linked to an incompatibility locus in Phalaris coerulescens with a recombination frequency of $11+0.03$ per cent (Leach \& Hayman, 1987). This linkage was first observed in Lolium species (Cornish et al., 1980., Fearon et al., 1983) who nominated the locus concerned as $S$. The original plants used in this study were homozygous for variants at this locus. Crosses were made between selfcompatible mutants and a related self-incompatible plant which has a heterozygote. Heterozygous hybrids were planted out in the field and selfed. Seedlings from selfing self-compatible hybrids were checked for their PGI-2 genotypes. A series of data indicative of linkage 
Table 3 The expected percentages of compatible pollen observed when a double mutant, including a mutant at a third unlinked locus $(T)$, is selfed and crossed as a pollen parent to the testers. The plants are assumed to be homozygous at $T$ before the mutation. A representative range of $S$ and $Z$ genotype are illustrated

\begin{tabular}{llllclc}
\hline Genotype & Selfing & $S_{12} Z_{12}$ & $S_{11} Z_{12}$ & $S_{22} Z_{12}$ & $S_{12} Z_{11}$ & $S_{12} Z_{22}$ \\
\hline$S_{12}^{*} Z_{12} T_{11}^{*}$ & 75 & 75 & 100 & 75 & 87.5 & 87.5 \\
$S_{11}^{*} Z_{12} T_{11}^{*}$ & 75 & 75 & 75 & 100 & 87.5 & 87.5 \\
$S_{11}^{*} Z_{11} T_{11}^{*}$ & 75 & 75 & 75 & 100 & 75 & 100 \\
\hline
\end{tabular}

Table 4 The stigmatic incompatibility phenotypes observed in 61 mutant plants

\begin{tabular}{|c|c|c|c|c|c|c|c|c|c|}
\hline & $S_{12} Z_{12}$ & $S_{12} Z_{11}$ & $S_{12} Z_{22}$ & $S_{11} Z_{12}$ & $S_{22} Z_{12}$ & $S_{11} Z_{11}$ & $S_{11} Z_{22}$ & $S_{22} Z_{11}$ & $S_{22} Z_{22}$ \\
\hline $\begin{array}{l}\text { Phenotype } \\
\text { frequency }\end{array}$ & 14 & 4 & 7 & 9 & 10 & 7 & 5 & 2 & 3 \\
\hline
\end{tabular}

Table 5 Disturbed segregations for PGI- 2 obtained in the selfed progeny of eight self-compatible mutants. The data are arranged for analysis so that the least frequent genotype is common to all families

\begin{tabular}{llll}
\hline Family & & & \\
\hline 1 & 51 & 64 & 5 \\
2 & 49 & 66 & 9 \\
3 & 63 & 46 & 5 \\
4 & 19 & 31 & 2 \\
5 & 40 & 47 & 5 \\
6 & 38 & 57 & 4 \\
7 & 44 & 77 & 6 \\
8 & 44 & 69 & 6 \\
\hline
\end{tabular}

Heterogeneity $\chi^{2}{ }_{14}=17.4,0.3>P>0.2$.

Recombination fraction is $11.4 \pm 0.14$.

are shown in Table 5. The linkage distance agrees with that calculated previously.

Mutants which showed linkage to GPI and which were heterozygous for $S$ were selfed and those homozygous for self-compatibility when their pollen was tested were homozygous for the $S$ allele concerned. GPI linkage alone may be used as an index of $S$ mutants. $S$ mutants were also analysed for linkage as indicated by disturbed segregations with further isozymic variants, including a leaf peroxidase (PER). No evidence of a disturbed segregation due to linkage was found with these genes. These mutants could be classified as pollen-only mutants when the stylar incompatibility phenotype showed the plant to be heterozygous at the $S$ locus.

Intercrosses made between many of the $S$ mutants gave plants which were 100 per cent self-compatible.
These crosses included some between the complete mutant and pollen-only mutants showing that they behaved as alleles.

\section{Identification of $\mathrm{Z}$ mutants}

Pollen-only $Z$ mutants may be recognized by reciprocal crosses with the testers when the mutant stylar genotype is heterozygous for $Z$ and the pollen phenotype is homozygous. Homozygotes obtained from such $Z$ heterozygotes are homozygous for the $Z$ locus concerned. No complete mutant at the $Z$ locus was identified.

$Z$ mutants showed no evidence of linkage with either PGI-2, a peroxidase gene, or any of the other isozymic variants tested, and were non-allelic in hybrids with $S$ mutants.

\section{Identification of a third class of mutants}

Evidence for the involvement of at least a third gene affecting incompatibility was provided by the following results. Self-compatible plants with the stylar genotype $S_{12} Z_{12}$ would be expected to carry a pollen-only mutant at either $S$ or $Z$ and on crossing to the testers as a male to give the results shown in Table 1 . Two such mutants failed to give these results and produced results like those shown in Table 2 . Thus the selfcompatibility mutant involved was not concerned with exclusive changes to either $S$ or $Z$ specificity. Selfed progeny of the mutants also gave disturbed segregations for isozymic variants at the leaf peroxidase locus PER.

Self-compatible plants with stigmatic genotypes heterozygous at either $S$ or $Z$ and homozygous at the 
other locus, and which contained a pollen only mutant at the heterozygous locus, would give clear evidence of this when used as a pollen parent on the testers (see Table 1). Heterozygotes for either $S$ or $Z$, which failed to show a pollen-only mutant at the heterozygous locus, would be expected to contain a self-compatible mutant at the homozygous locus if only $S$ and $Z$ mutants occurred. Families produced by selfing a number of self-compatible plants of this type also gave evidence of disturbed segregations for PER. A series of segregations illustrating this disturbance is shown in Table 6. This disturbance is explicable if the PER locus is closely linked to the self-compatible mutation. Families showing disturbed segregations for PER gave no evidence of disturbed segregations for PGI-2 or other isozymic variants.

Some homozygotes for the postulated PER-linked mutant were found to be heterozygous for $S$ or for $Z$, which indicates that a third gene is involved. A total of 16 PER-linked mutants has been identified. Homo-

Table 6 Disturbed segregations for a leaf peroxidase (PER) in the selfed progeny of 11 self-compatible mutants. The data were re-arranged for analysis so that the least frequent genotype was common to all families. Heterogeneity was tested for using a $G$-test for heterogeneity (Sokal \& Rohlf, 1981)

\begin{tabular}{llll}
\hline Family & & & \\
\hline 1 & 0 & 14 & 10 \\
2 & 4 & 56 & 41 \\
3 & 0 & 18 & 13 \\
4 & 0 & 15 & 12 \\
5 & 0 & 11 & 13 \\
6 & 1 & 10 & 13 \\
7 & 3 & 24 & 19 \\
8 & 1 & 31 & 37 \\
9 & 0 & 51 & 44 \\
10 & 0 & 56 & 53 \\
11 & 0 & 30 & 24 \\
\hline
\end{tabular}

Heterogeneity $\chi_{20}^{2}=23.74,0.5>P>0.3$. zygotes for eight of these have been obtained and they all behave as pollen-only mutants. The gene has been given the symbol $T$.

The evidence from the pollen grain ratios and from the absence of disturbed segregations at PGI-2 suggests that the $T$ gene is not closely linked to either $S$ or $Z$. Whether all $T$ mutants are allelic is not known as yet as crosses between them all have not been made.

Table 7 lists the results of the analysis of the selfcompatible mutants completed so far.

\section{Discussion}

The results from this study of mutants affecting incompatibility in Phalaris show considerable similarities to the results obtained in related studies reported in species with single locus gametophytic incompatibility systems. Mutants in which only the capacity to specify the pollen is modified are the most common ones detected in this study as reported in other studies. In Phalaris such mutants occur at both the $S$ and $Z$ loci and also at the postulated locus $T$. Mutants at which both stigma and pollen specificities are lost are also found in single locus systems and one such mutant has been identified at the $S$ locus in Phalaris.

In his significant and rather overlooked study of selfcompatible mutants in Secale, Lundqvist (1968) also recovered pollen-only mutants. He reports mutants at both the $S$ and $Z$ loci and remarks that the system he employed to identify and characterize the mutants could not identify mutants at a third (or and additional) locus. He indicates in his paper that such a class of mutants 'seems likely'.

A further point of similarity is the observation that more than half the seeds obtained from the mutant trap gave rise to plants which were both self-incompatible and also failed to give seed when crossed to $S_{12} Z_{12}$. Thus, these plants do not contain a functional incompatibility allele novel to those already present in the system. Lewis (1954) and others have reported similar observations and Lewis classified the seeds as resulting from 'revertants' involving a temporary loss of at least

Table 7 The self-compatible mutants analysed in this study which have been classified by some of the procedures described in the text. Mutants which it has not yet been possible to characterize as pollen-only or complete are described as 'unresolved'

\begin{tabular}{llllll}
\hline$S$ mutants & & $Z$ mutants & \multicolumn{2}{c}{$T$ mutants } \\
\hline$S_{1}$ pollen only & 5 & $Z_{1}$ pollen only & 1 & $T$ pollen only & 8 \\
$S_{2}$ pollen only & 5 & $Z_{2}$ pollen only & 5 & $T$ complete & 0 \\
$S$ complete & 1 & $Z$ complete & 0 & $T$ (unresolved) & 8 \\
$S$ (unresolved) & 5 & & & & \\
\hline
\end{tabular}


pollen specificity and its restoration in the next generation. The results from this study in Phalaris do not add anything to the understanding of such mutants. Nor does this study add anything to our knowledge of the origin of new functional incompatibility alleles. The extent of the study suggests that if these alleles arise at a single mutational step, then their rates of mutation must be very low indeed. This, too, agrees with the study of spontaneous mutation rate in Prunus (Lewis, 1948).

This similar spectrum of pollen-only and complete mutants in both single-locus and two-locus gametophytic systems probably reflects a basic similarity between the two systems in their genetic regulation of tissue specificity. This similarity may be quite independent of the precise chemical method of specification operating in the two systems. The difference in the effects of polyploidy in the two systems does indicate fundamental differences between them which are not simply explained by gene duplication (Lundqvist, 1974).

The difference in the number of $S$ and $Z$ mutants identified in this study, and the absence of $Z$ complete mutants, could well be a consequence of the relatively low number of mutants characterized, rather than providing critical evidence for a fundamental difference between the two genes. Lundqvist (1968) reported effectively equal numbers of $S$ and $Z$ mutants in this study and equal numbers of $S$ mutants for the two alleles tested. The unequal numbers of $Z$ mutants for the two alleles he tested probably reflects the unequal contribution of one allele to his study.

An unusual feature of this study is the demonstration that mutations at a gene other than $S$ or $Z$ can affect the incompatibility system. The evidence for at least a third gene being involved comes from three independent tests. Firstly, the fact that some selfincompatible mutants with a stigmatic genotype $S_{12} Z_{12}$ are not 100 per cent compatible with any of the testers when used as a male parent; secondly, that homozygotes for such mutants may be heterozygous or homozygous at either $S$ or $Z$; and thirdly that such mutants are apparently very closely linked to a gene specifying a leaf peroxidase. Such a linkage relationship is not shared by either $S$ or $Z$ mutants, which had been identified by their pollen compatibility on the testers.

To simplify the argument, consider this evidence to indicate the presence of only one additional gene $T$. Allelic variability for this gene does not appear to be concerned, as is variability at $S$ and $Z$, with the final incompatibility specification of the pollen grain. Genetic data obtained using other genotypes in this species (Hayman, 1956) show no evidence of genetic control of incompatibility by other than two genes. In all other gentically well-studied grass species, which include a wide range of temperate species, there is evidence for only two genes with multiple alleles involved in the incompatibility specification.

The $T$ mutants identified so far are all pollen-only mutants. However, the yield of complete mutants at $S$ and $Z$ was very low and this may well be a feature of the recovery of spontaneous mutants in the Poaceae. The absence of allelic variability at the $T$ locus means that a stigma-only mutant would not be self-compatible as a normal allele would be present, and such mutants would have escaped detection in this study. At the moment, there is no evidence from this study for any effect of the $T$ gene, or its mutants, on the incompatibility status of the stigma, and it may be that its action is limited to the pollen.

What is the role of the $T$ gene? Lundqvist and his colleagues have demonstrated that gametophytic incompatibility in Beta vulgaris and Ranunculus species is under the control of four non-allelic genes each with at least two alleles (Lundqvist, 1990a; Larsen, 1977; Osterbye, 1975). There is complementary interaction in specifying the pollen grain. It has been suggested that these dicotyledonous groups are representative of the condition existing among plants from which the monocotyledons arose (see Lundqvist, 1990a, for review). Possibly, then, the twolocus system present in the grasses may have been derived from a multi-locus system. On such an argument it could be suggested that allelic variability at the $T$ locus has become fixed, and in terms of specifying the pollen and style, it is a 'silent' and an invariant locus. It still performs a required function because mutants at this locus are recognizable because the pollen grain carrying them is self-compatible.

Fixation, however, has not been reported at any of the four loci in studies of a number of populations of Ranunculus (Osterbye, 1986; Lundqvist, 1990b). Furthermore, such an argument opens up the possibility that a single locus system could devolve from further fixation at either $S$ or $Z$. This has not been reported in any of the studies on incompatibility in the Poaceae. It is significant that none of the studies of mutation in single-locus gametophytic systems have reported ' $T$-like' mutants affecting self-incompatibility. The only mutants reported are at the $S$ locus, and no evidence has been reported for fixed incompatibility factors. It is also true that ' $T$-like' mutants closely linked to the $S$ locus may well escape detection, as they would behave as apparent $S$ mutants. Lewis (1979) has pointed out that a silent homozygous gene would explain the anomalous lack of allelic interaction in diploid pollen of Tradescantia which has a monogenic system. 
There are alternative arguments to account for the behaviour of $T$ mutants. Suppose the $T$ locus is thought of as having a function which is required to complete the formation of either any $S$ or, alternatively, any $Z$ gene product. It acts before $S$ or $Z$ specification and hence shows no $S$ or $Z$ allele specificity. A pollen grain carrying such a mutant forms none or imperfect $S$ or, alternatively, $Z$ products. If this is the situation there could be at least two types of additional genes for which self-compatible mutants would be recovered. One of these would be involved in processing what becomes an $S$ gene product and the other involved in processing what becomes a $Z$ gene product. Thus, on this model, the $T$ mutants act before specification and they may include at least two non-allelic groups.

A further possible basis for the $T$ mutants would be if they were to act after $S$ and $Z$ specification and to be involved in completing the interaction between $S$ and $Z$ products. The most likely basis for complementary interaction between $S$ and $Z$ gene products is the formation of a dimer and if the capacity to do this was under the control of the $T$ gene, then pollen grains carrying a mutant $T$ gene would be imperfectly specified irrespective of the $S$ or $Z$ alleles they carried.

Polyploid plants of Phalaris coerulescens are selfincompatible ( R. N. Oram, personal communication), as is found with other self-incompatible grasses. It would be anticipated that diploid pollen heterozygous for a $T$ mutant would be self-incompatible.

The implications of the existence of at least a third gene involved in the incompatibility system in the Poaceae suggests that genetic control of this system may be more complex than previously thought. It is interesting that the same possibility has been shown in the single locus sporophytic system found in the Cruciferae. Lewis et al. (1988) and Zuberi \& Lewis (1988) report genetic evidence for the presence of a gametophytically active gene with a low number of alleles in addition to the sporophytically acting $R$ gene in two species of Cruciferae.

Evidence for the presence of more than one locus related to incompatibility in the Cruciferae is also suggested by recent studies employing cDNA clones which encode $S$-specific glycoproteins in Brassica. DNA gel blot analysis of the Brassica genomic DNA has revealed the presence of a number of DNA sequences with varying degrees of homology to the $S$-locus specific glycoproteins. Trick \& Flavell (1989) and La Londe et al. (1989) each report on an expressed gene which is a member of this class of sequences. The genes are developmentally expressed in the stigma in the same manner as the $S$-locus specific gene and are not linked to the $S$ locus. La Londe et al. (1989) report that their sequence is present in self-compatible species.
Unlike the genes encoding $S$-locus specific glycoproteins, these genes show little sequence polymorphism among different $S$ genotypes. Boyes et al. (1991) report a second related gene linked to that reported by La Londe et al. (1989) which is also expressed in the stigma. Whether these genes are involved in the incompatibility recognition system or to pollination in general is not known.

\section{Acknowledgements}

We acknowledge financial support from the Australian Research Committee. The extensive field experiments were conducted at the South Australian Department of Agriculture Research Station at Northfield. We express our gratitude to the Manager and the staff for the use of this facility. We are grateful to Dr Carolyn Leach for her help and to Sharon Hutchins and Angela Binns for excellent technical assistance.

\section{References}

BOYES, D. C., CHEN, C.-H., TARTIKANJANA, T., ESK, J. J. AND NASRALLAH, J. B. 1991 . Isolation of a second $S$-locus-related cDNA from Brassica oleracea: Genetic relationships between the $S$-locus and two related loci. Genetics, 127, 221-228.

CORNISH, M., HAYWARD, M. D. AND LAWRENCE, M. J. 1980. Selfincompatibility in rye grass. III. The joint segregation of $S$ and PGI-2 in Lolium pereune L. Heredity, 44, 55-62.

FEARON, C. H., HAYWARD, M. D. AND LAWRENCE, M. J. 1983. Selfincompatibility in rye grass. V. Genetic control, linkage and seed set in diploid Lolium multiforum Lam. Heredity, 50, 35-46.

HAYMAN, D. L. 1956. The genetical control of incompatibility in Phalaris coerulescens. Aust. J. Biol. Sci, 9, 321-331.

LA LONDE, B. A., NASRAllah, M. E., DWYeR, K. G. CHEN, C.-H., BARLOW, B. AND NASRALlAH, J. B. 1989. A highly concerved Brassica gene with homology to the $S$-locus specific glycoprotein structural gene. The Plant Cell, 1, 249-258.

LARSEN, K. 1977. Self-incompatibility in Beta vulgaris L. I. Four gametophytic complementary $S$-loci in sugar beet. Hereditas, 85, 227-248.

LEACH, C. R. AND HAYMAN, D. L. 1987. The incompatibility loci as indicators of conserved linkage groups in the Poaceae. Heredity, 58, 303-305.

LEWIS, D. 1948. Structure of the incompatibility gene. I. Spontaneous mutation rate. Heredity, 2, 219-236.

LEWIS, D. 1951. Structure of the incompatibility gene. III. Types of spontaneous and induced mutations. Heredity, $\mathbf{5}$, 399-414.

LEWIS, D. 1954. Structure of the incompatibility gene. IV. Types of mutations in Prunus avium L. Heredity, 8, 357-363.

LEWIS, D. 1979. Genetic versatility of incompatibility in plants. New Zealand J. Bot. 17, 637-644. 
LEWIS, D. AND CROWE, L. 1953. Theory of revertible mutations. Nature (Lond.), 172, 501.

LEWIS, D., VERMA, S. C. AND ZUBERl, M. 1.1988. Gametophytic-sporophytic incompatibility in the Cruciferae - Rhaphanus sativus. Heredity, 61, 355-366.

LUNDQVisT. A. 1961. A rapid method for the analysis of incompatibility in grasses. Hereditas, 47, 705-707.

LUNDQVIST, A. 1962. The nature of the two-loci incompatibility system in grasses. I. The hypothesis of a duplicative origin. Hereditas, 48, 153-168.

LUNDQVIST, A. 1968. The mode of origin of self-fertility in grasses. Hereditas, 59, 413-426.

Lundovist, A. 1975. Complex self-incompatibility systems in angiosperms. Proc. R. Soc. Lond. B, 188, 235-245.

LUNDQVIST, A. 1990a. The complex $S$-gene system for control of self-incompatibility in the buttercup Ranunculus. Herditas, 113, 29-46.

LUNDQVIST, A. $1990 \mathrm{~b}$. Variability within and among populations in the 4-gene system for control of self-incom- patibility in Ranunculus polyanthemos. Hereditas, 113, 47-61.

OSTERBYE, U. 1975. Self-incompatibility in Ranunculus acris. I. Genetic interpretation and evolutionary aspects. Hereditas, 80, 91-112.

OSTERBYE, U. 1986. Self-incompatibility in Rananculus acris L. III. $S$-loci numbers and allelic identities. Hereditas, 104, 61-73.

SOKAL, R. R. AND ROHLF, F. J. 1981. Biometry: The Principles and Practice of Statistics in Biological Research, 2 nd edn. W. H. Freeman San Francisco.

TRICK, M. AND FlaVell, R. B. 1989. A homozygous $S$ genotype of Brassica oleraceae expresses two $S$-like genes. Mol. Gen. Genet., 218, 112-117.

WATKINS, A. E. 1925. Genetical and cytological studies in wheat. J. Genet, 15, 323-366.

ZUBERI, M. I. AND LEWIS, D. 1988. Gametophytic-sporophytic incompatibility in Cruciferae - Brassica campestris. Heredity, 61, 367-377. 